\title{
The interfering effect of serial cognitive structures in long-term memory on word-order in short-term memory ${ }^{1}$
}

DONALD L. MOSHER, University of Connecticut, Storrs, Conn. 06268

This experiment investigated the retention of word-position information, independent of item information, using a distractor STM paradigm. Triads of words, which formed a serial cognitive structure presumably in LTM, were presented to 192 $S$ in natural or scrambled orders with serial-, backwards-, or free-recall instructions. Triads selected from different taxonomic classes were presented on the eight trials to ensure high item retention. Sine half of the Ss made no item errors at all, their responses were scored for retention of word-position information. There were few position errors with the natural ordered triads with either serial- or backswards-recall instructions. There was a build-up of position errors across trials for the scrambled order-serial recall group. The scrambled order-backwards recall group had a high rate of position errors beginning on Trial 1. With free-recall instructions, the scrambled triads were transposed frequently back to their natural serial order. These data are incompatible with features of some box models of memory.

The present experiment concerned itself with the influence of previously learned cognitive structures on proactive interference (PI) in short-term memory (STM). The focus of the study was on the influence of previously learned serial orders, viewed as cognitive structures, on order errors in STM. Loess's $(1967,1968)$ research indicated that the recall of word triads will reveal little PI when a new taxonomic class is presented on each trial. If the word triads drawn from different taxonomic classes are congruent with serial cognitive structures in long-term memory (e.g., nickel, dime, quarter, or Tuesday, Wednesday, Thursday), few errors in recalling the words in their correct serial order are to be expected. However, if the word triads deviate from the previously learned serial orders (e.g., dime, nickel, quarter, or Thursday, Tuesday, Wednesday), then the words all may be retrieved, but errors in the recall of the position of the items are to be expected. With backwards recall instructions the task should remain relatively simple for the natural serial orders, but it becomes much more complex for the serial orders which are incongruent with previous learning outside the experimental situation. Free-recall could range from zero to three for any word triad. A 2 by 3 by 8 analysis of variance with repeated measures on the trials factor was computed. As expected, there were relatively few recall errors, the mean recall for all groups and trials being 2.83 words. The analysis revealed a significant triple interaction . $(\mathrm{F}=2.39 ; \mathrm{df}=14,1302$; $\mathrm{p}<.01)$, which appeared to be uninterpretable. The only other significant $F$ ratio was for the main effect of trials $(\mathrm{F}=3.66 ; \mathrm{df}=7,1302 ; \mathrm{p}<.001)$, which can be interpreted as showing an irregular decline from a mean recall of 2.98 on Trial 1 to 2.75 on Trial 8 , with the largest decline appearing from Trial 1 to Trial 2, which had a mean of 2.88 words recalled.

The purpose of changing taxonomic classes with each trial was to ensure a high level of word recall since the variable of major import to be examined was transposition of word order. Examination of the word-recall scores revealed that exactly half of the $128 \mathrm{Ss}$ in the serial-backwards by ordered-unordered groups-had recalled all triads correctly on each of the eight trials. Furthermore, these Ss were distributed 15, 17,16 , and 16 among the four groups. To equalize the $\mathrm{N}, 15 \mathrm{Ss}$ from each group were selected randomly, and their protocols were rescored for correct word position. Since all of these Ss had recalled all 24 of the words presented in the eight trials, it was possible to assign one point for each correct word position independently of the number of words recalled which was constant. The independent word-position scores could range from zero to three. Figure 1 presents the mean word position for the four groups across the eight trials.

An analysis of variance for correct word position yielded seven highly significant $F$ ratios as follows: Triads (A), F $(1,56)$ : 39.67; Instructions (b) $\mathrm{F}(1,56)=7.78$; A $x$ B, F $(1,56)-8.13$; Trials (C), F $(7,1188)=6.23 ; A \times C, F(7,1188)=5.30$; $\mathrm{B} \times \mathrm{C}, \mathrm{F}(7,1188)=6.33 ; \mathrm{A} \times \mathrm{B} \times \mathrm{C}, \mathrm{F}=$ 21.86 .

The results can be summarized verbally by pointing out that there were almost no word-position errors for the natural-order triads with either the serial or backwards recall instructions. The serial recall-unordered triads group correctly positioned the words on the first two trials at a near-errorless level, which was comparable to the two ordered triads groups, then they showed a rapid build-up in errors across the next two trials, some improvement for two trials, and another decline over the last two trials. Their mean correct word position declined from 2.87 for the first two trials to a mean of 2.50 for the last two trials although the curve did not decrease in a simple monotonic fashion. The backwards recall-unordered triads group had great difficulty with word position on 


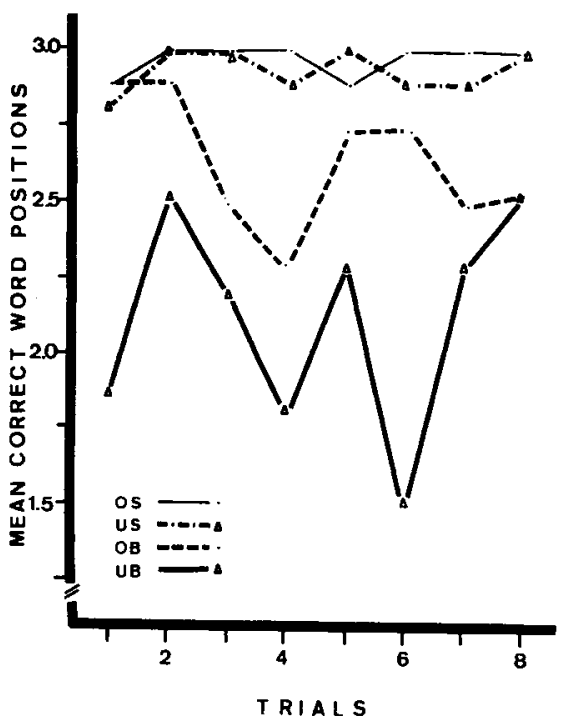

Fig. 1. Retention of word-position information for the ordered-serial (OS), unordered-serial (US), ordered-backwards (OB), and unordered-backwards (UB) groups.

Trial 1 with a mean of 1.87 words correctly positioned. Their performance varied in a saw-tooth fashion across trials with no simple trend toward more or less errors across trials. The serial-ordered group had an overall mean of 2.97 ; the backwards-ordered group had an overall mean of 2.93; the serial-unordered group's mean was 2.61 ; and the backwards-unordered mean was 2.12 .

The data from the free-recall/unorderedtriads group was analyzed to determine the Ss' tendency to return to the natural serial orders from the scrambled serial orders. Frequencies of recall in the presented order and the natural order (other orders were excluded from consideration) for the free-recall/unordered-triads group and the serial-recall/unordered-triads group were compared by a chi squared analysis. In the free-recall condition, the $S$ s reverted to the natural order in 19 of 204 instances $\left(\chi^{2}=5.20 ;\right.$ df $\left.=1 ; \quad p<.05\right)$. A second comparison, using the free-recall/unorderedtriads data, examined the occurrence of all word positions other than the presented scrambled orders. As stated above, the Ss recalled the triads in the natural order on 88 occasions and they used orders other than the presented or natural order on 38 occasions. A highly significant chi square $\left(\chi^{2}=19.44 ; \mathrm{df}=1 ; \mathrm{p}<.001\right)$ was found when comparing the occurrence of the natural and the other orders against chance expectations. These analyses indicated that Ss do tend to employ the natural serial orders as a retrieval aid when not constrained by instructions.

\section{DISCUSSION}

These data highlight the importance of making a distinction between the retrieval of words and the retrieval of word positions in STM. In this experiment, which changed taxonomic classes on each trial, the serial-, backwards-, and free-recall instructions exerted no simple effect on the recall of the words. Presenting the words in their natural serial sequence or in a scrambled sequence likewise had no simple effect on the S's ability to recall the words. Word recall was uniformly good in this experiment since $94 \%$ of the words presented were recalled correctly after the 15 -sec interval filled with the counting-backwards task.

The small number of errors in word retrieval permitted an analysis of the errors in word position which was independent of word recall. Since half of the Ss made no error in word recall across the eight trials, it was possible to examine errors in word position independently of errors in words recalled. It was anticipated that errors in word position should be minimal when the words were presented in their natural serial order. This indeed was the case, and the backwards-recall instructions did not appreciably alter the difficulty of the task above the level of the serial-order instructions. It is not difficult to transform an ascending or well-learned serial order in to a descending or backwards order. However, the retrieval of correct word position was more difficult when the triads were presented in a scrambled or nonnatural order. The serial-unordered group made few errors on the first two trials and then began to show a decline which, while not a simple monotonic function, still suggested a build-up in interference. Two types of interference were possible in this situation. On Trial 1 the presented nonnatural order could be interfered with by the better established natural order presumably in a LTM store. On succeeding trials, in addition to the interference from the natural order (1-2-3), interference could begin between the various presented permutations of nonnatural orders, e.g., Order $2-1-3$ is followed by Order 1-3-2 by Order 2-3-1, etc. With the serial-unordered group, the decline in accuracy of word position began to manifest itself with a large decline on Trial 3, suggesting the importance of the latter type of scrambled permutation interference. The backwards-unordered group, however, showed a large immediate deficit on Trial 1. Their information-processing capacity was greatly taxed, even on the first trial, when required to retain unordered triads during $15 \mathrm{sec}$, distracted by counting backwards, and then to give the correct backwards order. Item retention was unaffected, but word-position information was lost.

The data from the free-recall/unordered group supported the contention that the natural serial orders exert an influence on retrieval of word position. When not constrained by serial instructions, the free-recall group transposed the presented scrambled orders into the natural orders at a much greater-than-chance level.

The present experiment demonstrated the importance and feasibility of distinguishing between item information and position information in STM. When item information was recalled with complete accuracy, it was possible, by selecting triads of words with a natural serial order and presenting them in a scrambled serial order, to demonstrate interference in the retrieval of position information and that $\mathrm{Ss}$ revert to retrieving the words in their natural serial order when not constrained by instructions. These data, then, like those of Murdock \& vom Saal (1967), cast doubt on the generality of Conrad's (1965) model of order errors in immediate recall. Conrad argued that what appeared to be order errors are often item errors, and he presented some data to support a "box" model, which assumed input into a fixed sequence of storage locations and a decline in signal-to-noise ratios of the input across time, making acoustic substitutions at recall more probable. The present data indicate that position information can be lost while item information is retained. Also, these data are incompatible with simple flow-chart or box models indicating unidirectional transmission from STM to LTM storage. As Norman (1968) has recently argued, interconnections between storage systems for primary and secondary memory must be so direct and complete that formal distinctions between the two systems are difficult to make. The data from this experiment clearly indicate that serial structures in LTM play some role in disrupting the recall of item position in unordered triads in the STM paradigm, thus suggesting an interconnection of some kind.

\section{REFERENCES}

BROWN, J. Some tests of the decay theory of immediate memory. Quarterly Journal of Experimental Psychology, 1958, 10, 12-21.

CONRAD, R. Order errors in immediate recall of sequences. Journal of Verbal Learning \& Verbal Behavior, 1965, 4, 161-169.

LOESS, H. Short-term memory, word class, and sequence of items. Journal of Experimental Psychology, 1967, 74, 556-561.

LOESS, H. Short-term memory and item similarity. Journal of Verbal Learning \& Verbal Behavior, 1968, 7, 87-92.

MURDOCK, B. B., JR. Distractor and probe techniques in short-term memory. Canadian Journal of Psychology, 1967, 27, 316-324.

MURDOCK, B. B., JR., \& vom SAAL, W. 
Transpositions in short-term memory. Journal of Experimental Psychology, 1967, 74, 137-143.

NORMAN, D. A. Toward a theory of memory and attention. Psychological Review, 1968, 75, 522-536.

PETERSON, D. R., \& PETERSON, M. J. Short-term retention of individual verbal items. Journal of Experimental Psychology, 1959, 58 , 192-198.
NOTE

1. I wish to thank Mrs. Edith V. Sullivan for he assistance in collecting and analyzing the data and Dr. Michael T. Turvey for his comments upon the the manuscript and for stimulating my in terest in short-term memory. This research was supported by a grant from the University of Connecticut Research Foundation.

\section{Effect of simultaneous presentation of conceptually similar and dissimilar items on paired associate learning ${ }^{1}$}

\begin{abstract}
PAUL S. SHERMAN and SAM C. BROWN, Kansas State University, Manhattan, Kans. 66502
\end{abstract}

Paired-associate learning was retarded significantly when subsets of conceptually dissimilar pairs were presented simultaneously rather than successively for learning. The retardation was eliminated, however, when the subsets contained pairs sharing similar stimulus terms or similar response terms. Under both methods of presentation, learning was faster when pairs were grouped on the basis of conceptual similarity than when the same pairs were presented in dissimilar groupings.

The purpose of the present research was to determine the effect on paired-associate (PA) learning of (a) blocking similar and dissimilar subsets of pairs within the list for sequential presentation, and (b) presenting pairs within each block either successively (one at a time) or simultaneously (all together) for learning. Previous research (Brown \& Brown, 1965; Brown \& Read, 1966) showed that simultaneous presentation of more than one pair enabled Ss to categorize the pairs into subsets, but that such categorization retarded PA learning significantly when compared with the standard, successive method of pair presentation. In the earlier research, conceptually unrelated items were blocked together for simultaneous presentation. Research by Rotberg \& Woolman (1963) has shown that blocked, successive presentation of related materials produced better PA performance than unblocked presentation. On the basis of this finding, it was predicted that the deleterious effect of simultaneous presentation found earlier should be reduced or eliminated if the blocking of pairs were based on some organizational principle. Consequently, the present study compared simultaneous and successive methods of pair presentation under conditions where pairs grouped for contiguous presentation were conceptually similar or dissimilar. To obtain more general information on the effects of locus of similarity, pairs were grouped on the basis of stimulus or response term similarity.

\section{METHOD}

The 128 college student Ss practiced on an identical list of 16 paired associates for 20 trials. The list was exactly the list used previously by Underwood \& Schultz (1961; List 5). The 16 stimulus (S) terms consisted of four categories of four words each. Each category represented a different conceptual class of words (male first names, animals, dances, and clergymen). The 16 response (R) members represented instances of four different categories of four words each (diseases, sports, countries, and chemical elements). The pairs were constructed by pairing the S-terms in each conceptual category with R-terms from different categories (e.g., Bob-Nitrogen; Bill-Sparrow; Joe-Germany; John-Cancer).
Learning for all Ss was carried out under the recall (pairing-test) procedure. Four of the eight groups of $16 \mathrm{Ss}$ each learned under a successive method (individual pair presentation on pairing and test series) and four under a simultaneous method (blocks of four pairs grouped together for simultaneous exposure on pairing and test series). The four simultaneous groups differed with respect to the kind of pair groupings used (conceptually organized or unorganized). For Group SO (S-term organization), the four pairs within each block contained $S$ terms from the same conceptual category and $R$ terms from different categories. With Group RO (R-term organization), each block of four pairs consisted of $R$ terms from the same category and $S$ terms from different categories. Within each list ( $S O$ and $R O$ ), the same pairs were grouped together on each trial. The two remaining simultaneous groups learned unorganized blocks of pairs, i.e., each block of four pairs presented for simultaneous exposure contained $S$ and $R$ terms from different conceptual categories. For Group UC (unorganized-constant grouping), the same four dissimilar pairs were grouped together on every trial, following the procedure for Groups SO and RO. In Group UV (unorganized-varied grouping), the pairs within each block changed from trial to trial. Thus, conceptual organization was varied by using four lists of the same $S-R$ pairs which differed only in the temporal order of presentation of these pairs in the list.

Under the successive method of pair presentation, each of four groups learned exactly one of the four list orders used by each of the simultaneous groups. Consequently, the pairs were blocked in the same way under both methods of pair presentation. However, with the successive method, the pairs within each block were exposed individually.

The list members were presented on slides either individually (16 slides) or vertically in blocks of four (4 slides) under the successive and simultaneous methods, respectively. Corresponding slides were produced for S-terms. A group-data collection procedure was used wherein all $16 \mathrm{Ss}$ in each group served in a single session. Each trial began with the successive presentation of the 4 or 16 slides containing the pairs, through a Kodak slide projector, followed by the successive exposure of corresponding slides containing the S-terms, during which Ss were asked to write down in answer booklets the correct R-term for each S-term. Rate of presentation of the slides on the pairing part of each trial was 2 and $8 \mathrm{sec}$, and on test series, 4 and $16 \mathrm{sec}$ for successive and simultaneous groups, respectively. The interval between pairing-test series and trials 